\title{
The Concentration of Digoxin Activity after Intravenous in Three Compartments Mathematical Model
}

\author{
Lakshmi Narayan. K, Siva Rama Krishna Reddy. V
}

\begin{abstract}
Present paper is designed to compare the distribution of digoxin in three compartment model administered through an intravenous (i.v). These models under consideration is denoted by a system of non-linear ordinary differential equations. The Eigenvalue and the Laplace transform methods were used to solve the system of equations. Digoxin was administered to five subjects through Intravenous then, the serum digoxin concentrations were measured respectively over a period of 72 hours. The transfer coefficients were obtained from observed digoxin concentrations using method of residuals and the variation of digoxin concentration - time curves plotted using MATLAB.
\end{abstract}

Index Terms: Digoxin, Blood plasma, Intestinal fluid, Cells, Laplace transform, Eigenvalue,

\section{INTRODUCTION}

The elucidation of the simplest pharmacokinetic model compatible with the data observed when prescribing drugs in humans is a necessary step to understand the distribution, elimination, activity and toxicity of the drug. This is becoming increasingly important with low therapeutic index drugs and serious side effects such as cardiac digoxin glycoside.

The purpose of this study was to perform an experiment in human designed to compare adjustments of an open pharmacokinetic model to two and three compartments with experimentally determined time-serum digoxin concentration data.

Previous research reported in the literature [1-2] graphical analysis of the blood concentration of digoxin versus time after intravenous administration in humans has shown that a semi-logarithmic plot can be divided into two or three exponential segments, the choice being dependent on blood sampling intervals and graphic techniques used. These analyzes also imply that a two or three compartment model can best describe the temporal evolution of digoxin in humans. In this study, we used the same set of experimental data (Table 3.2) to fit the three compartment model. The transfer coefficients were obtained from observed digoxin concentrations (Table 3.4) using method of residuals and the variation of digoxin concentration - time curves plotted in two and three compartment models of each subject (Table 3.1) using MATLAB.

Revised Version Manuscript Received on 16 September, 2019 Lakshmi Narayan. $\mathbf{K}^{\mathbf{1}}$, Department of Mathematics, Vidya Jyothi Institute of Technology, Hyderabad, India.

Siva Rama Krishna Reddy. $\mathbf{V}^{\mathbf{2}}$, Department of Mathematics, Gokaraju Rangaraju Institute of Engineering and Technology, Hyderabad, India.

\section{THREE COMPARTMENT MODEL}

In the present model, we consider a three compartment catenary model in which the first compartment is connected to the second compartment and the second compartment is connected to the third compartment, a single dosage of drug flow via first compartment to second compartment, also from second compartment to third compartment and the elimination occurs from last compartment. The first compartment corresponds to blood plasma then from there the drug diffuses into the second compartment namely intestinal fluid and finally it enters into third compartment of Cells as shown in Figure 2.1.

Let $x_{1}(t), x_{2}(t)$ and $x_{3}(t)$ denote the amount of drug in blood plasma compartment, Intestinal fluid compartment and Cells compartment respectively.

Let $k_{011}$ be the initial amount of drug dosage. The mathematical structure of the three compartment system describing the drug administration is given by the set of ordinary differential equations as follows:

$$
\begin{aligned}
& \frac{d x_{1}(t)}{d t}=-k_{12} x_{1}+k_{21} x_{2^{s}} \quad x_{1}(0)=k_{01} \\
& \frac{d x_{2}(t)}{d t}=k_{12} x_{1}-\left(k_{21}+k_{23}\right) x_{2}+k_{22} x_{2} ; x_{2}(0)=0 \\
& \frac{d x_{2}(t)}{d t}=k_{2 \mathrm{a}} x_{2}-\left(k_{22}+k_{20}\right) x_{2} ; \quad x_{2}(0)=0
\end{aligned}
$$

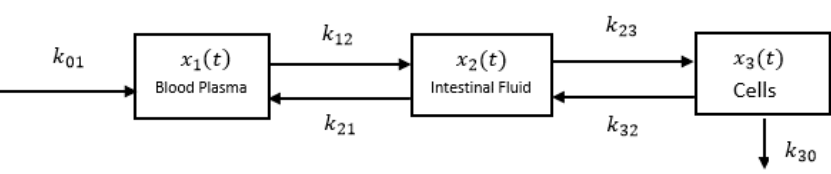

Fig 2.1 General scheme of the three compartment model Each equation describes the change in drug concentration in their respective compartments over time. Also the quantities $k_{12}$ and $k_{21} ; k_{23}$ and $k_{32}$ denote the rate constants from blood plasma compartment to intestinal fluid compartment; intestinal fluid compartment to cells compartment and vice-versa respectively and $k_{30}$ denote the clearance constants from cell compartment only. 


\section{The Concentration of Digoxin Activity after Intravenous in Three Compartments Mathematical Model}

In matrix notation of (1), we have

$$
\frac{d X}{d t}=K X
$$

Where

$X=\left[\begin{array}{l}x_{1}(t) \\ x_{2}(t) \\ x_{3}(t)\end{array}\right] \quad$ and $\quad K=\left[\begin{array}{ccc}-k_{12} & k_{21} & 0 \\ k_{12} & -\left(k_{21}+k_{23}\right) & k_{32} \\ 0 & k_{23} & -\left(k_{32}+k_{20}\right)\end{array}\right]$

The Laplace transform [3] - [4] is an integral transform where the linear operator $\mathcal{L}\{f(t)\}$ transform a function $f(t)$ with $t \in \mathbb{R}$ from the time domain to a function $F(s)$ with $s \in \mathbb{C}$ in an image domain. The advantage of this transformation is that differentiation and integration in the time domain corresponds to simple algebraic operations in the image domain.

Applying the Laplace transform to (4) gives,

$$
\begin{aligned}
\mathcal{L}\left\{X^{s}(t)\right\} & =\mathcal{L}\{K X\} \Leftrightarrow s \mathcal{L}\{X(t)\}-X(0)=K \mathcal{L}\{X(t)\} \\
& \Leftrightarrow(s I-K) \mathcal{L}\{X(t)\}=X(0) \\
& \Leftrightarrow L(s) . \mathcal{L}\{X(t)\}=X(0)
\end{aligned}
$$

Where,

$L(s)=\left[\begin{array}{ccc}s+k_{12} & -k_{21} & 0 \\ -k_{12} & s+\left(k_{21}+k_{23}\right) & -k_{32} \\ 0 & -k_{23} & s+\left(k_{32}+k_{20}\right)\end{array}\right]$

We solve the system of equations (6) by Cramer's rule.

$$
\begin{aligned}
& \operatorname{det}(L(s))=\operatorname{det}(s I-K) \\
& =\left(s+\lambda_{1}\right)\left(s+\lambda_{2}\right)\left(s+\lambda_{2}\right)
\end{aligned}
$$

Where,

$$
\begin{aligned}
& \lambda_{1}+\lambda_{2}+\lambda_{2}=k_{20}+k_{12}+k_{21}+k_{23}+k_{32} \\
& \lambda_{1} \lambda_{2} \lambda_{a}=k_{12}\left(k_{22}+k_{23}\right)+k_{21}\left(k_{22}+k_{20}\right)+k_{20}\left(k_{2 a}+k_{12}\right) \\
& \lambda_{1} \lambda_{2}+\lambda_{2} \lambda_{3}+\lambda_{3} \lambda_{1}=k_{12} k_{23} k_{20}
\end{aligned}
$$

To calculate the solution of the central compartment $x_{1}(t)$, peripheral compartment $x_{2}(t)$ and cells compartment $x_{\mathrm{a}}(t)$, we substitute the vector $X(0)$ into the first column, second column and third column of the matrix $L(s)$ and denote the resulting matrices by $L_{1}(s), L_{2}(s)$ and $L_{g}(s)$ respectively, the quotient from Cramer's rule, we get

$$
\begin{aligned}
& \mathcal{L}\left\{x_{1}(t)\right\}=\frac{\operatorname{det}\left(L_{1}(s)\right)}{\operatorname{det}(L(s))} \\
& \mathcal{L}\left\{x_{2}(t)\right\}=\frac{\operatorname{det}\left(L_{2}(s)\right)}{\operatorname{det}(L(s))}
\end{aligned}
$$

$$
\mathcal{L}\left\{x_{\mathrm{a}}(t)\right\}=\frac{\operatorname{det}\left(L_{a}(s)\right)}{\operatorname{det}(L(s))}
$$

Now, from (5) - (10), the Laplace back transform is

$$
\begin{aligned}
& x_{1}(t)=\mathcal{L}^{-1}\left\{\frac{k_{01}\left[\left(s+\left(k_{21}+k_{23}\right)\right)\left(s+\left(k_{22}+k_{20}\right)\right)-k_{23} k_{22}\right]}{\left(s+\lambda_{1}\right)\left(s+\lambda_{2}\right)\left(s+\lambda_{2}\right)}\right\} \\
& x_{2}(t)=\mathcal{L}^{-1}\left\{\frac{k_{01} k_{12}\left(s+k_{32}+k_{20}\right)}{\left(s+\lambda_{1}\right)\left(s+\lambda_{2}\right)\left(s+\lambda_{9}\right)}\right\} \\
& x_{9}(t)=\mathcal{L}^{-1}\left\{\frac{k_{01} k_{12} k_{23}}{\left(s+\lambda_{1}\right)\left(s+\lambda_{2}\right)\left(s+\lambda_{2}\right)}\right\}
\end{aligned}
$$

By applying Heaviside's theorem, from equation (11), (12) and (13) we get.

$$
\begin{aligned}
& x_{1}(t)= \\
& \frac{k_{01}\left\{\left(k_{21}+k_{23}-\lambda_{1}\right)\left(k_{32}+k_{20}-\lambda_{1}\right)-k_{2 a} k_{32}\right\}}{\left(\lambda_{2}-\lambda_{1}\right)\left(\lambda_{3}-\lambda_{1}\right)} e^{-\lambda_{1} t} \\
& +\frac{k_{01}\left\{\left(k_{21}+k_{23}-\lambda_{2}\right)\left(k_{32}+k_{20}-\lambda_{2}\right)-k_{23} k_{32}\right\}}{\left(\lambda_{2}-\lambda_{2}\right)\left(\lambda_{1}-\lambda_{2}\right)} e^{-\lambda_{2} t} \\
& +\frac{k_{01}\left\{\left(k_{21}+k_{23}-\lambda_{3}\right)\left(k_{32}+k_{20}-\lambda_{92}\right)-k_{23} k_{32}\right\}}{\left(\lambda_{1}-\lambda_{3}\right)\left(\lambda_{2}-\lambda_{2}\right)} e^{-\lambda_{2} t} \\
& x_{2}(t)=\frac{k_{01} k_{12}\left(k_{20}+k_{32}-\lambda_{1}\right)}{\left(\lambda_{3}-\lambda_{1}\right)\left(\lambda_{2}-\lambda_{1}\right)} e^{-\lambda_{1} t} \\
& \begin{aligned}
&+ \frac{k_{01} k_{12}\left(k_{20}+k_{32}-\lambda_{2}\right)}{\left(\lambda_{33}-\lambda_{2}\right)\left(\lambda_{1}-\lambda_{2}\right)} e^{-\lambda_{2} t} \\
&+\frac{k_{01} k_{12}\left(k_{20}+k_{32}-\lambda_{2}\right)}{\left(\lambda_{1}-\lambda_{3}\right)\left(\lambda_{2}-\lambda_{2}\right)} e^{-\lambda_{3} t}
\end{aligned} \\
& x_{\mathrm{g}}(t)=\frac{k_{01} k_{12} k_{23}}{\left(\lambda_{3}-\lambda_{1}\right)\left(\lambda_{2}-\lambda_{1}\right)} e^{-\lambda_{1} t} \\
& +\frac{k_{01} k_{12} k_{23}}{\left(\lambda_{13}-\lambda_{2}\right)\left(\lambda_{31}-\lambda_{1}\right)} e^{-\lambda_{2} t} \\
& +\frac{k_{01} k_{12} k_{23}}{\left(\lambda_{1}-\lambda_{2}\right)\left(\lambda_{1}-\lambda_{3}\right)} e^{-\lambda_{3} t}
\end{aligned}
$$

\section{CLINICAL APPLICATION}

Table 3.1: Patients Characteristics of the Subjects

\begin{tabular}{|c|c|c|c|}
\hline Subject & $\begin{array}{c}\text { Age } \\
\text { (years) }\end{array}$ & $\begin{array}{c}\text { Height } \\
\text { (inches) }\end{array}$ & $\begin{array}{c}\text { Weight } \\
\text { (kilograms) }\end{array}$ \\
\hline C.C. & 24 & 70 & 72.7 \\
\hline T.F. & 26 & 70 & 72.7 \\
\hline C.J. & 24 & 71 & 75 \\
\hline W.S. & 28 & 70 & 77.3 \\
\hline D.L. & 23 & 75 & 85.5 \\
\hline
\end{tabular}


*Table 3.2: Experimentally Observed Digoxin Concentrations (ng/ml) by Subjects

\begin{tabular}{|c|c|c|c|c|c|c|c|c|c|}
\hline \multicolumn{2}{|c|}{ C.C. } & \multicolumn{2}{c|}{ T.F. } & \multicolumn{2}{c|}{ C.J. } & \multicolumn{2}{c|}{ W.S. } & \multicolumn{2}{c|}{ D.L. } \\
\hline \begin{tabular}{|c|c|c|c|c|c|c|c|c|} 
Time \\
$(\mathbf{m i n})$
\end{tabular} & Digoxin & $\begin{array}{l}\text { Time } \\
(\mathbf{m i n})\end{array}$ & Digoxin & $\begin{array}{l}\text { Time } \\
(\mathbf{m i n})\end{array}$ & Digoxin & $\begin{array}{l}\text { Time } \\
(\mathbf{m i n})\end{array}$ & Digoxin & $\begin{array}{l}\text { Time } \\
(\mathbf{m i n})\end{array}$ & Digoxin \\
\hline 2.4 & 21 & 2.4 & 22.5 & 2.1 & 26.5 & 2.5 & 22 & 2.1 & 20.5 \\
\hline 4.3 & 22 & 4.1 & 21 & 4.1 & 21 & 4.5 & 18 & 4.1 & 17.5 \\
\hline 6.2 & 18 & 6.2 & 19 & 6 & 18.8 & 6.1 & 20.5 & 6.1 & 14.5 \\
\hline 8.2 & 15 & 8.2 & 18.5 & 8.1 & 16.5 & 8.1 & 18.5 & 8.1 & 12.5 \\
\hline 10.2 & 15.5 & 10.3 & 16.8 & 10.1 & 14.3 & 10.1 & 16.5 & 10.1 & 13 \\
\hline 14.2 & 14 & 14.2 & 15.8 & 14.1 & 14 & 14.1 & 16.5 & 14.1 & 12 \\
\hline 18.2 & 11.3 & 18.2 & 14.5 & 18.1 & 13 & 18.3 & 11.5 & 18.1 & 11 \\
\hline 22.2 & 13.5 & 22.1 & 14 & 22.1 & 12.3 & 22.6 & 13 & 22.1 & 9.1 \\
\hline 30.1 & 12.5 & 30.2 & 13.3 & 30 & 10.3 & 30.4 & 9.8 & 30.1 & 9.6 \\
\hline 45.2 & 9.1 & 45.4 & 9.5 & 45.1 & 7.6 & 45.3 & 7.3 & 45.2 & 5.6 \\
\hline 60.2 & 6.7 & 61.5 & 7.3 & 60.6 & 5.8 & 60.2 & 5.5 & 60.2 & 4.9 \\
\hline 120.7 & 3.6 & 120.1 & 3.6 & 120 & 3.3 & 121 & 2.8 & 120.3 & 3.2 \\
\hline 185.8 & 2.6 & 182 & 2.3 & 183 & 2.2 & 182 & 2 & 180.5 & 2 \\
\hline 234.8 & 2.2 & 233 & 1.8 & 210 & 1.9 & 230 & 1.4 & 241.8 & 1.8 \\
\hline 355.3 & 1.2 & 356 & 1.2 & 351 & 0.52 & 351 & 0.73 & ---- & --- \\
\hline 485 & 1.05 & 467 & 1.1 & 469 & 0.57 & 480 & 0.55 & 470 & 0.9 \\
\hline 944 & 0.75 & 943 & 1.03 & ---- & ----- & 924 & 0.5 & 948 & 0.85 \\
\hline 1429 & 1 & 1465 & 0.93 & 1426 & 0.5 & ----- & ---- & 1423 & 0.7 \\
\hline 2618 & 0.54 & 2119 & 0.53 & 2082 & 0.52 & 2080 & 0.4 & 2187 & 0.45 \\
\hline 2869 & 0.63 & 2854 & 0.44 & 2833 & 0.33 & 2842 & 0.36 & 2831 & 0.43 \\
\hline 4306 & 0.53 & 4301 & 0.27 & 4305 & 0.24 & 4320 & 0.16 & 4305 & 0.39 \\
\hline
\end{tabular}

* From [5]

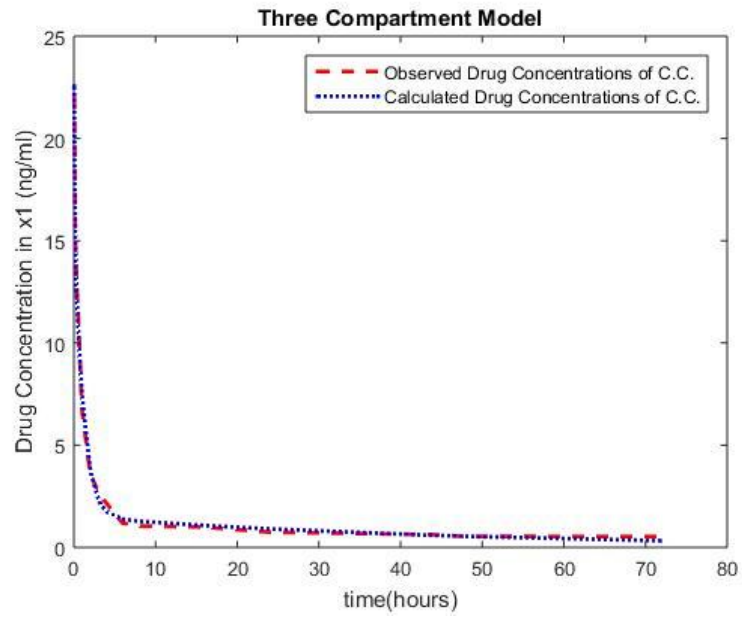

Fig 3.1: Plot of Observed (dashed line) and Calculated Digoxin (dotted line) Concentrations - time profiles of C.C. in three-compartment model.

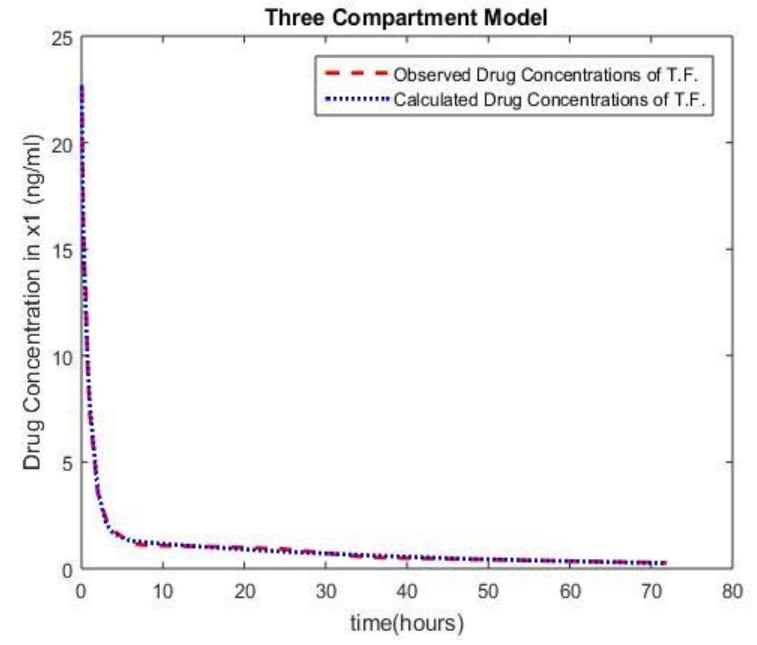

Fig 3.2: Plot of Observed (dashed line) and Calculated (dotted line) Digoxin Concentrations - time profiles of T.F. in three-compartment model 


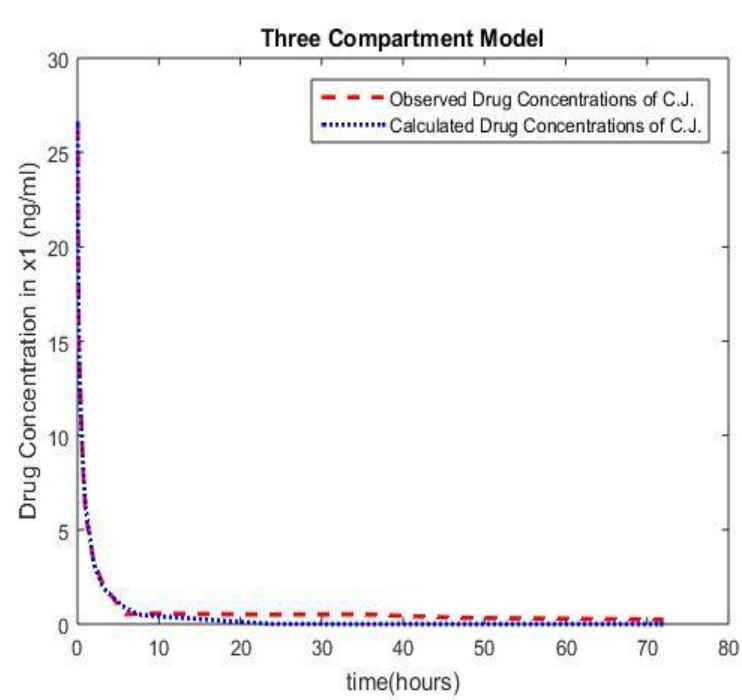

Fig 3.3: Plot of Observed (dashed line) and Calculated (dotted line) Digoxin Concentrations - time profiles of C.J. in three-compartment model

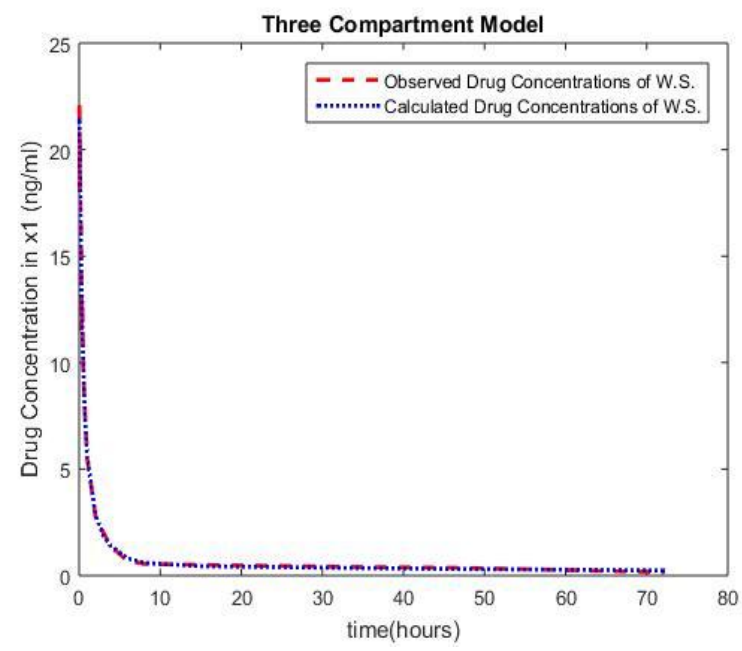

Fig 3.4: Plot of Observed (dashed line) and Calculated (dotted line) Digoxin Concentrations - time profiles of W.S. in three-compartment model

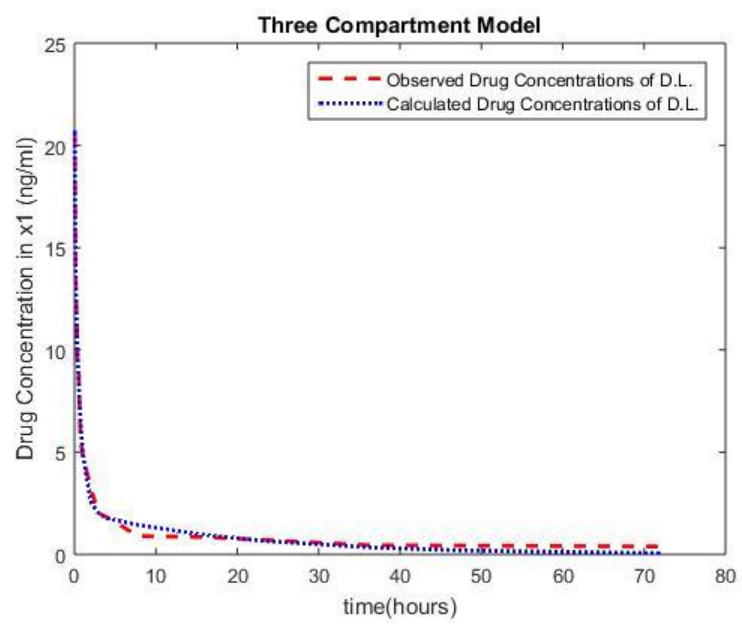

Fig 3.5: Plot of Observed (dashed line) and Calculated (dotted line) Digoxin Concentrations - time profiles of D.L. three-compartment model

\begin{tabular}{|c|c|}
\hline Subject & RMSE Values \\
\hline C.C. & 0.4637 \\
\hline T.F. & 0.3109 \\
\hline C.J. & 0.3765 \\
\hline W.S. & 0.333 \\
\hline D.L. & 0.3729 \\
\hline
\end{tabular}

Table 3.3: RMSE values of three compartment models of each Subject

\begin{tabular}{|c|c|c|c|c|c|}
\hline $\begin{array}{c}\text { Subject } \\
\text { Name }\end{array}$ & $\boldsymbol{k}_{\mathbf{1 2}}$ & $\boldsymbol{k}_{\mathbf{2 1}}$ & $\boldsymbol{k}_{\mathbf{2 3}}$ & $\boldsymbol{k}_{\mathbf{3 2}}$ & $\boldsymbol{k}_{\mathbf{3 0}}$ \\
\hline C.C. & 0.4344 & 1.2322 & 0.4993 & 0.0213 & 0.0077 \\
\hline T.F. & 0.5712 & 1.1934 & 0.6654 & 0.0389 & 0.1035 \\
\hline C.J. & 0.7444 & 1.2045 & 0.5923 & 0.0473 & 0.0198 \\
\hline W.S. & 0.4179 & 1.7235 & 0.6432 & 0.0312 & 0.0117 \\
\hline D.L. & 0.4964 & 1.3765 & 0.5723 & 0.0195 & 0.0231 \\
\hline
\end{tabular}

Table 3.4: Parameter values of three compartment model of each Subject

\section{CONCLUSION}

In this present investigation, we formulated a three compartment model using a coupled ordinary differential equations. The results obtained from our model are coincides with clinical data with RMSE $<0.5$ (Table 3.3) which is considered as an efficient model for the data. We observed that our model gives more accurate results for three compartmental model. The solution curves for clinical data and model were drawn using MATLAB and it was observed that the results (Figure 3.1-3.5) were almost coincides. So we can predict serum digoxin concentrations for any given time using our model.

\section{REFERENCES}

1. R. H. Reuning, R. A. Sams, and R. E. Notari. Role of pharmacokinetics in drug dosage adjustment, i. Pharmacologic effect kinetics and apparent volume of distribution of digoxin. J. Clin. Pharmacol. 13:127-141 (1973).

2. J.E. Doherty, W. H. Perkins, and G. K. Mitchell. Tritiated digoxin studies in human subjects. Arch. Int. Med. 108:87-95 (1961) 9

3. David Vernon Widder. The Laplace Transform. Princeton University Press, 1966

4. G. Doetsch. Einführung in Theorie und Anwendung der Laplace-Transformation. Birkhäuser Verlag, 1976.

5. William G.Kramer, Richard P. Lewis et.al. Pharmacokinetics of Digoxin: Comparison of a two and a three compartment model in Man, Journal of Pharmacokinetics and Biopharmaceutics, Vol 2, No. 4, 1974.

6. R. E. Notari. Biopharmaceutics and Pharmacokinetics, an Introduction, Dekker, New York, 1971, pp. 124-130.

7. J. G. Wagner. Biopharrnaceutics and Relevant Pharmacokinetics, Drug Intelligence Publications, Hamilton, Ill., 1971, p. 295.

8. J. E. Doherty, C. B. Ferrell, and E. J. Towbin. Localization of the renal excretion of tritiated digoxin. Am. J. Med. Sci. 258:181 189 (1969).

9. L. Z. Benet. General treatment of linear mammillary models with elimination from any compartment as used in pharmacokinetics. J. Pharm. Sci. 61:536-541 (1972).

10. R. C. Weast (ed.). Handbook of Tables for Mathematics, 4th ed. Chemical Rubber Co., Columbus, O., 1970, p. 130.

11. T. W. Smith, V. P. Butler, Jr., and E. Haber. Determination of therapeutic and toxic serum digoxin concentrations by radioimmunoassay. New Eng. J. Med. 281:1212-1216 (1969). 
12. F. I. Marcus, L. Burkhalter, C. Cuccia, J. Pavlovich, and G. G. Kapadia. Administration of digoxin with and without a loading dose. Circulation 34:865-874 (1966).

13. F. I. Marcus, G. J. Kapadia, and G. G. Kapadia. The metabolism of digoxin in normal subjects. J. PharmacoL Exp. Ther. 145:203-209 (1964).

\section{AUTHORS PROFILE}

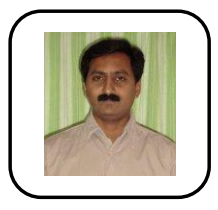

Dr. Lakshmi Narayan K completed M.Sc Applied Mathematics from Osmania University, Hyderabad, $\mathrm{Ph}$. D from JNTUH Hyderabad. He has 24 years teaching experience. Presently he is working as Professor of Mathematics in Vidya Jyothi Institute of Technology Hyderabad. His area of research is Mathematical Modeling like Mathematical Ecology, Epidemiology and Pharmacokinetics. Under his guidance 6 Ph.D scholars awarded, two are submitted their thesis and $4 \mathrm{M}$. Phil Scholars were awarded. He was published more than 100 research articles in reputed National/International Journal and he was attended and presented his research articles in more than $40 \mathrm{National/International} \mathrm{conferences.}$

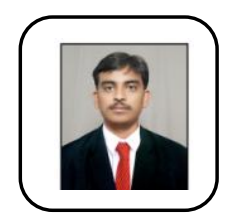

Mr. Siva Rama Krishna Reddy. V, born in 1982, is an Assistant Professor in Mathematics. He presently holds the appointment at Gokaraju Rangaraju Institute of Engineering and Technology, Hyderabad, Telengana. He obtained M. Sc., from Kakatiya University, Warangal, in 2005. He has more than 9 years of teaching experience. He is pursuing Ph. D from Rayalaseema University, Kurnool, in the areas of "Bio-Mathematical Modeling". He has recently communicated some papers in his area of interest. 